Original article

\title{
Effect of local modulation in enzymatic homeostasis on bone turnover marker dynamics in blood at substituting femur defects with vaterite scaffolds
}

\author{
Aleksey N. Ivanov, Yuliya A. Chibrikova, Mariia S. Saveleva, Vladimir V. Ostrovskij, Igor A. Norkin \\ Saratov State Medical University, Saratov, Russia
}

Received 3 July 2020, Revised 28 October 2020, Accepted 4 November 2020

(C) 2020, Ivanov A.N., Chibrikova Yu.A., Saveleva M.S., Ostrovskij V.V., Norkin I.A.

(C) 2020, Russian Open Medical Journal

\begin{abstract}
The goal of this research was the investigation of concentration changes in the blood bone turnover markers during local modulation of enzymatic homeostasis by means of targeted delivery of alkaline phosphatase (ALP) with polycaprolactone (PCL) and vaterite (VT) scaffolds implanted into the femur defects in white rats.

Material and Methods - The tests of PCL/VT/ALP scaffold implantations into the bone defects were performed on 30 white rats, and the serum of intact animals was used as the control. ELISA and multiplex assay were used to find inflammatory and bone turnover markers including monocyte chemoattractant-1, sclerostin, fibroblast growth factor-23, connective tissue growth factor (CTGF), osteoprotegerin, osteocalcin, $\beta$-cross laps and the activity of tartrate-resistant acid phosphatase- $5 \mathrm{~b}$ in the blood of experimental animals. The activity of serum ALP was tested with the conventional kinetic method. The morphology of the reparative processes was verified by microscopy of specimens taken from the implantation areas and stained with hematoxylin or eosin.

Results - The PCL/VT/ALP scaffold implantations into the bone defects of white rats caused active osteogenesis along with the steady rise in osteocalcin concentration in blood. ALP activity in the blood did not depend on the exogenous enzyme in the scaffold and rose by the 28th day after the implantations. The targeted ALP delivery into the defect area caused the rise in CTGF concentration as well as the decrease in blood sclerostin within a short time after the implantations.

Conclusion - The modulation of the local enzyme homeostasis by means of the targeted ALP delivery with PCL/VT scaffolds stimulated reparative osteogenesis within a short time after the implantations with no changes to the bloodstream or local inflammatory changes suggesting their biocompatibility and the safety in use.
\end{abstract}

Keywords: scaffold, regeneration, bone turnover markers.

Cite as Ivanov AN, Chibrikova YuA, Saveleva MS, Ostrovskij VV, Norkin IA. Effect of local modulation in enzymatic homeostasis on bone turnover marker dynamics in blood at substituting femur defects with vaterite scaffolds. Russian Open Medical Journal 2020; 9: e0414.

Correspondence to Aleksey N. Ivanov. Address: 148, N.G. Chernyshevskogo str., Saratov, 410002, Russian Federation. Phone: +7927279959. E-mail: lex558452@gmail.com.

\section{Introduction}

The new prospects of solving the tenacious problem of bone turnover stimulation are associated with the elaboration of scaffolds capable of structural and functional substitution of bone intercellular matrix and acceleration of reparative osteogenesis. Scaffolds for bone turnover stimulation should possess certain osteoconductive mechanical properties, and therefore they are often made of synthetic polymers that have a long biodegradation time [1]. Polycaprolactone (PCL) is one of the biocompatible polymers that feature long biodegradation time and physical integrity, it is commonly used in biomedical technologies, and for bone turnover stimulating scaffolds in particular [1-3]. Their osteoinductive properties are enhanced with mineral components - phosphate and carbonate calcium compounds [3, 4]. Hydroxyapatite, tricalcium phosphate, and other calcium phosphate salts are the most frequent options for this purpose [3, $5,6]$. The mineralization of scaffold fibers with vaterite (VT) looks promising as it stimulates the proliferation of osteoblasts, easily transforms into hydroxyapatite during bone tissue mineralization and thanks to its porous surface is capable of adsorbing various functional substances and releasing them into the body after implantation when recrystallized into calcite, providing targeted delivery of these compounds locally in the defect area $[7,8]$.

The alkaline phosphatase (ALP) gene is one of the first to be expressed during calcification suggesting the pivotal role of this enzyme for early stages of mineralization [9]. Therefore, local modulation of enzymatic homeostasis in the area of the bone defect by means of targeted ALP delivery with scaffolds brings in new prospects for stimulation of bone turnover regeneration. In this regard, the purpose of this work was the investigation of changes in concentrations of bone metabolism markers in blood at local modulation of enzymatic homeostasis by means of targeted ALP delivery with PCL/VT-scaffolds implanted into femur defects in white rats. 
Table 1. Dynamics of osteoblastogenesis markers after the implantations of scaffolds in the animals of the comparison and the experimental groups

\begin{tabular}{|c|c|c|c|c|c|}
\hline \multirow{2}{*}{ Indicators } & \multirow{2}{*}{$\begin{array}{c}\text { Controls } \\
(n=10)\end{array}$} & \multicolumn{2}{|c|}{ Comparison group } & \multicolumn{2}{|c|}{ Experimental group } \\
\hline & & 7 days $(n=7)$ & 28 days $(n=8)$ & 7 days $(n=7)$ & 28 days $(n=8)$ \\
\hline \multirow{2}{*}{ CTGF, ng/ml } & \multirow{2}{*}{$0(0,1.39)$} & \multirow{2}{*}{$1.95(1.33,5.06)$} & $1.51(1.26,3)$ & $5.9(3.74,13)$ & $2.56(1.07,8.1)$ \\
\hline & & & $p_{1}=0.045, p_{2}=0.417$ & $p_{1}<0.001, p_{3}=0.032$ & $p_{1}=0.032, p_{2}=0.127, p_{3}=0.713$ \\
\hline \multirow{2}{*}{ FGF23, $\mathrm{pg} / \mathrm{ml}$} & \multirow{2}{*}{$248(172,403)$} & $337(226,617)$ & $263(196,272)$ & $465(379,507)$ & $361(299,537)$ \\
\hline & & $\mathrm{p}_{1}=0.204$ & $\mathrm{p}_{1}=0.093, \mathrm{p}_{2}=0.266381$ & $p_{1}=0.056, p_{3}=562$ & $p_{1}=0.197, p_{2}=0.318, p_{3}=0.052$ \\
\hline \multirow{2}{*}{ SOST, $\mathrm{pg} / \mathrm{ml}$} & \multirow{2}{*}{$310(221,544)$} & $527(411,649)$ & $318(184,425)$ & $316(210,367)$ & $220(169,301)$ \\
\hline & & $\mathrm{p}_{1}=0.097$ & $p_{1}=0.722, p_{2}=0.017$ & $p_{1}=0.625, p_{3}=0.001$ & $p_{1}=0.197, p_{2}=0.270, p_{3}=0.318$ \\
\hline \multirow{2}{*}{$\mathrm{OC}, \mathrm{ng} / \mathrm{ml}$} & \multirow{2}{*}{$75.6(47.7,91)$} & $267(220,320)$ & $255(224,300)$ & $271(233,521)$ & $306(219,393)$ \\
\hline & & $\mathrm{p}_{1}=0.005$ & $\mathrm{p}_{1}=0.002, \mathrm{p}_{2}=0.846$ & $p_{1}=0.008, p_{3}=0.648$ & $p_{1}=0.003 p_{2}=0.871 p_{3}=0.271$ \\
\hline \multirow{2}{*}{$A L P, u / I$} & \multirow{2}{*}{$191(139,222)$} & $182(169,211)$ & $362(292,455)$ & $211(184,228)$ & $322(285,365)$ \\
\hline & & $\mathrm{p}_{1}=0.751$ & $\mathrm{p}_{1}<0.001, \mathrm{p}_{2}<0.001$ & $\mathrm{p}_{1}=0.214, \mathrm{p}_{3}=0.099$ & $\mathrm{p}_{1}<0.001, \mathrm{p}_{2}<0.001, \mathrm{p}_{3}<0.052$ \\
\hline
\end{tabular}

Data presented as median with lower and upper quartiles - Me (LQ, UQ). $\mathrm{p}_{1,2,3}$ - statistical significance of differences as compared to the controls, parameters of the group on E7 after the implantations and the comparison group within the same time after the surgical interventions.

Table 2. Dynamics of osteoclastogenesis markers after the implantations of scaffolds in the animals of the comparison and the experimental groups

\begin{tabular}{|c|c|c|c|c|c|}
\hline \multirow{2}{*}{ Indicators } & \multirow{2}{*}{$\begin{array}{c}\text { Controls } \\
(n=10)\end{array}$} & \multicolumn{2}{|c|}{ Comparison group } & \multicolumn{2}{|c|}{ Experimental group } \\
\hline & & 7 days $(n=7)$ & 28 days $(n=8)$ & 7 days $(n=7)$ & 28 days $(n=8)$ \\
\hline \multirow{4}{*}{$\begin{array}{l}\mathrm{MCP}-1 \mathrm{ng} / \mathrm{ml} \\
\mathrm{OPG}, \mathrm{pg} / \mathrm{ml}\end{array}$} & 2.2 & $2.5(2.3,2.8)$ & $2.6(2.1,3.1)$ & $2.2(2,2.4)$ & $2.1(0.9,3.2)$ \\
\hline & $(1.9,28)$ & $p_{1}=0.406$ & $p_{1}=0.888, p_{2}=0.862$ & $\mathrm{p}_{1}=0.858, \mathrm{p}_{3}=0.093$ & $p_{1}=0.755, p_{2}=0.874, p_{3}=0.462$ \\
\hline & 926 & $981(721,1118)$ & $603(423,891)$ & $538(461,1232)$ & $643(499,1203)$ \\
\hline & $(426,1219)$ & $p_{1}=0.883$ & $p_{1}=0.450, p_{2}=0.093$ & $\mathrm{p}_{1}=0.824, \mathrm{p}_{3}=0.271$ & $\mathrm{p}_{1}=0.824, \mathrm{p}_{2}=0.874, \mathrm{p}_{3}=0.636$ \\
\hline \multirow{4}{*}{$\begin{array}{l}\text { TRACP, u/l } \\
\text { CTX-I, ng/ml }\end{array}$} & 0.4 & $1.06(0.37,2.04)$ & $0.42(0.29,0.81)$ & $1.06(0.71,1.5)$ & $0.43(0.3,0.72)$ \\
\hline & $(0.21,0.41)$ & $\mathrm{p}_{1}=0.028$ & $\mathrm{p}_{1}=0.182, \mathrm{p}_{2}=0.049$ & $\mathrm{p}_{1}<0.001, \mathrm{p}_{3}=0.999$ & $\mathrm{p}_{1}=0.182, \mathrm{p}_{2}=0.023, \mathrm{p}_{3}=0.833$ \\
\hline & 9.7 & $23.7(10.9,26.2)$ & $19.3(9.5,29.7)$ & $9.6(8.7,15.8)$ & $10.57(7.5,16.3)$ \\
\hline & $(6.6,21.2)$ & $\mathrm{p}_{1}=0.087$ & $\mathrm{p}_{1}=0.168, \mathrm{p}_{2}=0.907$ & $\mathrm{p}_{1}=0.088, \mathrm{p}_{3}=0.049$ & $\mathrm{p}_{1}=0.893, \mathrm{p}_{2}=0.674, \mathrm{p}_{3}=0.189$ \\
\hline
\end{tabular}

$\mathrm{p}_{1,2,3}$ - statistical significance of differences as compared to the controls, parameters of the group on E7 after the implantations and the comparison group within the same time after the surgical interventions.

\section{Material and Methods}

\section{Animals}

The experiment involved 40 male white rats and was conducted in compliance with the Declaration of Helsinki as well as the recommendations of the Ethics Committee of the Federal State Budgetary Educational Institution of Higher Education 'V.I. Razumovsky Saratov State Medical University', the Russian Federation Ministry of Healthcare (Minutes No. 6 of February 06, 2018). The rats were anaesthetized with $0.1 \mathrm{ml} / \mathrm{kg}$ of Telazol (Zoetis Inc., USA) $+0.1 \mathrm{ml} / \mathrm{kg}$ of Xylanite (Nita-Farm, Russia) intramuscular injections before the procedures.

The animals were divided into three groups: the control group included 10 intact animals, the comparison group included 15 rats that had PCL/VT scaffolds implanted into them, and the experimental group included 15 rats that had PCL/VT scaffolds with the adsorbed ALP implanted into them. The PCL matrixes were electrospun and mineralized with vaterite by the procedure presented in [7]. ALP (Sigma-Aldrich Chemie, Germany) adsorption was performed by scaffold immersion in the $4 \mathrm{mg} / \mathrm{ml}$ ALP enzyme solution, then rinsed in deionized water and drained in a drying oven. The matrixes were implanted into longitudinal defects of femur bones shaped like $2 \times$ 5-7 mm long and 2-3 mm deep linear notches. Blood samples were collected by means of cardiac puncture of the animals of both the comparison and the experimental groups on Day 7/E7 (n=7) and Day 28/E28 (n=8) after the implantations. The animals were then sacrificed by anesthetic overdosage.

\section{Biochemical tests}

The connective tissue growth factor (CTGF), monocyte chemoattractant protein-1 (MCP-1), fibroblast growth factor-23
(FGF23), osteoprotegerin (OPG) and sclerostin (SOST) concentrations in blood were measured using a multiplex assay with MagPix (Luminex, USA) system, Rat Vascular Injury Panel 1 and Rat Bone Panel 1 (Merck Millipore, USA) under the instructions attached to the reagent kits. ELISA was used to find concentrations of osteocalcin $(\mathrm{OC})$ in serum, and C-terminal telopeptide of type I collagen (CTX-I), Rat-MIDTM RatTRAPTM, RatLAPSTM (IDS, UK) panels, and microplate spectrophotometer Anthos 2020 (Biochrom, UK) in conformity with the manufacturers' instructions to find tartrate-resistant acid phosphatase-5b (TRACP) activity. ALP serum activity was found using Sapphire-400 (Hirose Electronic System, Japan) analyzer, and DiaSys (Germany) reagents.

\section{Histology tests}

The morphological verification of the reparative processes was carried out on femur bones retrieved from animals of both the experimental $(n=8)$ and the comparison $(n=8)$ groups sacrificed on E28. The cross-sections of the femur shafts with scaffold implantation sections were obtained by means of standard procedure and stained with hematoxylin and eosin (BioVitrum, Russia). The microscopy of the specimens was performed with Axiolmager Z2 (CarlZeiss, Germany). The qualitative assessment of trabeculae and vessels in the scaffolds as well as their integration with the edges of cortical plate defects was performed at microscopy.

\section{Statistical analysis}

Statistica 10.0 software was used for statistical data processing. The calculation of the Shapiro-Wilk test showed the absence of normal distribution for the findings, and therefore they 
were represented as a medial value, lower and upper quartiles Me (LQ, UQ). The Mann-Whitney test was used to compare the groups. The differences were considered significant at $p<0.05$.

\section{Results}

It was found that CTGF concentrations in blood of the experimental animals with ALP scaffolds implanted into them on E7 were significantly higher than those in rats of the comparison group (see Table 1). CTGF concentrations in the experimental group surpassed the control values on E28 but did not differ from those of the comparison group (Table 1). SOST concentrations in the comparison group tended to rise regarding the controls on E7, but significantly dropped by E28 (Table 1). SOST concentration in the serum of the animals with ALP scaffolds implanted into them was significantly lower than in the rats of the comparison group on E7. OC concentrations in the blood of the experimental and comparison animals did not differ and exceeded the control values 3.3-4-fold on both E7 and E28. In animals of the experimental group the trend for the increase in OC level regarding the comparison group was observed on E28 (Table 1), which however never reached the statistical significance. ALP activity in the serum of animals of both the experimental and the comparison groups on E7 after scaffold implantations did not differ from that of the controls, but by E28 significantly increased by 1.7-1.9-fold (Table $1)$.

No significant changes in the concentration of either MCP-1 or OPG was found in the experimental animals (Table 2) while in the serum of animals of both the experimental and the comparison groups the increase of TRACP activity was found on E7; it returned to the norm on E28 (Table 2). CTX-1 level in animals of both the experimental and the comparison groups laid within the variability limits across all times of observation. However, on E7 this parameter was significantly lower in rats that had ALP scaffolds implanted into them (Table 2).

No signs of inflammation were found in animals of both the comparison and the experimental groups by E28 in the implantation areas though morphological examination. In the animals of the experimental group the scaffolds and the edges of cortical defects were tightly united with trabeculae, and covered the defects totally in 75 percent of the cases, while in the comparison group the integrations of two scaffold sides with the edges of cortical plate defect was observed in 50 percent of the cases. The areas of the implanted PCL/VT and PCL/VT/ALP scaffolds were mostly covered with anastomosing trabeculae, and fibroblastic cells as well as thin-walled vessels were found in the gaps between them.

Osteointegration with the edges of the cortical plate defects and trabeculae formations in PCL/VT and PCL/VT/ALP scaffolds proved their colonization with osteoblasts. The revealed changes in regulator and activity marker concentrations in blood of the animals of both the experimental and the comparison groups are therefore associated with osteogenesis proceeding in the scaffolds. The absence of local inflammation signs at implantations of the tested scaffolds accounted for the normal level of MCP-1 chemokine in the serum of both the comparison and experimental animals.

\section{Discussion}

The findings of the research suggested that the targeted ALP delivery to the defect areas with PCL/VT scaffolds increased CTGF serum concentration within a short time after the implantation. According to the published studies, CTGF enhances the intensity of mineralization and osteoblast proliferation [10], and therefore its more pronounced concentration in the blood of the experimental animals indicates the stimulating effect of the ALP targeted delivery to osteogenesis induction. However the analysis of the factors inhibiting osteoblastogenesis and mineralization (SOST and FGF23) [11, 12] in the blood of the experimental animals suggested the absence of the inhibiting effect on osteoblast activity in the tested scaffolds. Since SOST production by osteocytes is mostly defined with the mechanical loading [11], its decrease in blood of the comparison animals by E28 featured the recovery of the extremity supporting function. As the serum SOST in the animals that had scaffolds with ALP implanted into them was significantly lower than that in the comparison rats on E7, the recovery of the extremity biomechanics presumably proceeded quicker. The dynamics of osteoblast synthetic activity and mineralization markers (OC and ALP) in the controls and the comparison animals had no relevant differences and featured the increase in osteoblastogenesis activity. The absence of any significant increase in serum ALP activity on E7 suggested the effect of the enzyme retained in the scaffold being mostly local with no pronounced diffusion into systemic blood flow.

The research revealed no significant changes in concentrations of either MCP-1 or OPG that regulated osteoclastogenesis according to the published studies [13]. However TRACP activity rose in the blood of both the comparison and experimental animals on E7 suggesting the increase in the osteoclast activity [14]. As CTX-1 (a marker of organic bone matrix turnover) concentrations didn't change [14] after the implantations of $\mathrm{PCL} / \mathrm{VT}$ scaffolds into the femur defects, the increase in osteoclast activity within a short time after the implantations featured the turnover of the scaffold mineral component rather than the bone tissue.

Osteointegration with the edges of the cortical defect and trabeculae formation in both PCL/VT and PCL/VT/ALP scaffolds proved their populating with osteoblasts. Therefore, the observed changes in concentrations of osteoblast regulators and activity markers in the blood of experimental and comparison animals were associated with osteogenesis proceeding inside the scaffolds. The absence of signs for local inflammation in implantations of the tested scaffolds was accounted for by the normal content of MCP1 chemokine in the serum of both the comparison and experimental animals.

Therefore, osteogenesis actively proceeded in PCL/VT and PCL/VT/ALP scaffolds after their implantations into the femur bone defects featuring their osteoinductive effects biochemically presented with the increased concentrations of the growth factors that stimulated osteoblasts as well as markers of their synthetic activity in blood. The activation of osteoclastogenesis within a short time after the implantations was mainly aimed at the remodeling of the scaffold mineral component without the rise in organic component cleavage in the bone tissue.

\section{Conclusion}

The modulation of local fermentative homeostasis by means of ALP targeted delivery with PCL/VT scaffolds proceeds without 
changes in either serum activity of the enzyme or local inflammatory changes suggesting their biocompatibility as well as safety in use. ALP targeted delivery with PCL/VT scaffolds into the femur bone defect area stimulates reparative osteogenesis due to the increase in CTGF production as well as the decrease of SOST expression within a short time after implantation.

\section{Ethical approval}

All procedures of this study were performed in compliance with the Declaration of Helsinki, as well as recommendations of the Ethics Committee of the Saratov State Medical University (Minutes No. 6 of February 06, 2018)

\section{Funding}

The research was performed under the Government Assignment to Research Institute of Traumatology, Orthopedics and Neurosurgery of Saratov Saratov Medical University 'Designing technologies for the assessment of regeneration potential in matrixes for bone tissue defect replacements based on their vascularization parameters', Reg. No. AAAAA18-118020290178-3.

\section{Conflict of interest}

We have no conflict of interest to declare.

\section{References}

1. Dwivedi R, Kumar S, Pandey R, Mahajan A, Nandana D, Katti DS, et al. Polycaprolactone as biomaterial for bone scaffolds: review of literature. J Oral Biol Craniofac Res 2020; 10(1): 381-388. https://doi.org/10.1016/i.jobcr.2019.10.003.

2. Ivanov AN, Saveleva MS, Kurtukova MO, Kustodov SV, Gladkova EV, Blinnikova VV, et al. Particularities of bone regeneration in rats after implantation of polycaprolactone scaffold mineralized with vaterite with adsorbed tannic acid. Bull Exp Biol Med 2019; 167(2): 275-278. https://doi.org/10.1007/s10517-019-04508-x.

3. Andrade TM, Mello DCR, Elias CMV, Abdala JMA, Silva E, Vasconcellos $\mathrm{LMR}$, et al. In vitro and in vivo evaluation of rotary-jet-spun poly ( $\varepsilon$ caprolactone) with high loading of nano-hydroxyapatite. J Mater Sci Mater Med 2019; 30(2): 19. https://doi.org/10.1007/s10856-0196222-1.

4. Donate R, Monzón M, Ortega Z, Wang L, Ribeiro V, Pestana D, et al. Comparison between calcium carbonate and $\beta$-tricalcium phosphate as additives of 3D printed scaffolds with polylactic acid matrix. J Tissue Eng Regen Med 2020; 14(2): 272-283. https://doi.org/10.1002/term.2990.

5. Ivanov AN, Saveleva MS, Kozadaev MN, Matveeva OV, Sal'kovskiy YuE, Lyubun GP, et al. New approaches to scaffold biocompatibility assessment. BioNanoSci 2019; 9(2): 395-405 https://doi.org/10.1007/s12668-019-00613-3.

6. Rogowska-Tylman J, Locs J, Salma I, Woźniak B, Pilmane M, Zalite V, et al. In vivo and in vitro study of a novel nanohydroxyapatite sonocoated scaffolds for enhanced bone regeneration. Mater Sci Eng C Mater Biol App/ 2019; 99: 669-684. https://doi.org/10.1016/j.msec.2019.01.084.

7. Saveleva MS, Ivanov AN, Kurtukova MO, Atkin VS, Ivanova A, Lyubun $\mathrm{GP}$, et al. Hybrid $\mathrm{PCL} / \mathrm{CaCO}_{3}$ scaffolds with capabilities of carrying biologically active molecules. Mater Sci Eng C Mater Biol Appl 2018; 85: 57-67. https://doi.org/10.1016/i.msec.2017.12.019.

8. Stengelin E, Kuzmina A, Beltramo GL, Koziol MF, Besch L, Schröder R, et al. Bone Scaffolds Based on Degradable Vaterite/PEG-Composite Microgels. Adv Healthc Mater 2020; 9(11): e1901820. https://doi.org/10.1002/adhm.201901820.

9. Hendesi H, Barbe MF, Safadi FF, Monroy MA, Popoff SN. Integrin mediated adhesion of osteoblasts to connective tissue growth factor (CTGF/CCN2) induces cytoskeleton reorganization and cell differentiation. PLoS One 2015; 10(2): e0115325. https://doi.org/10.1371/journal.pone.0115325.

10. Millán JL. The role of phosphatases in the initiation of skeletal mineralization. Calcif Tissue Int 2013; 93(4): 299-306. https://doi.org/10.1007\%2Fs00223-012-9672-8.

11. Galea GL, Lanyon LE, Price JS. Sclerostin's role in bone's adaptive response to mechanical loading. Bone 2017; 96: 38-44. https://doi.org/10.1016\%2Fj.bone.2016.10.008.

12. Guo YC, Yuan Q. Fibroblast growth factor 23 and bone mineralization. Int J Oral Sci 2015; 7(1): 8-13. https://doi.org/10.1038\%2Fijos.2015.1.

13. Mulholland BS, Forwood MR, Morrison NA. Monocyte chemoattractant protein-1 (MCP-1/CCL2) drives activation of bone remodelling and skeletal metastasis. Curr Osteoporos Rep 2019; 17(6): 538-547. https://doi.org/10.1007/s11914-019-00545-7.

14. Vervloet MG, Brandenburg VM; CKD-MBD working group of ERA-EDTA. Circulating markers of bone turnover. J Nephrol 2017; 30(5): 663-670. https://doi.org/10.1007/s40620-017-0408-8.

\section{Authors:}

Aleksey N. Ivanov - MD, DSc, Head of the Laboratory Diagnostics Deaprtment, Research Institute of Traumatology, Orthopedics and Neurosurgery, Saratov State Medical University, Saratov, Russia; Central Scientific Research Laboratory, FSBEI HE I.V. Razumovsky Saratov SMU MOH Russia, Saratov, Russia. https://orcid.org/0000-0003-4061-5221.

Yuliya A. Chibrikova - Resident Doctor, Research Institute of Traumatology, Orthopedics and Neurosurgery, Saratov State Medical University, Saratov, Russia. https://orcid.org/0000-0001-8376-1099.

Mariia S. Saveleva - Junior Research Assitant, Central Research Laboratory, Saratov State Medical University, Saratov, Russia. https://orcid.org/0000-0003-2021-0462.

Vladimir V. Ostrovskij - MD, DSc, Director of Research Institute of Traumatology, Orthopedics and Neurosurgery, Saratov State Medical University, Saratov, Russia. https://orcid.org/0000-0002-8602-2715.

Igor A. Norkin - MD, DSc, Deputy Director for Development, Research Institute of Traumatology, Orthopedics and Neurosurgery, Saratov State Medical University, Saratov, Russia. https://orcid.org/0000-0002-6770$\underline{3398 .}$. 